\title{
SOME REMARKS ABOUT ADDITIVE AND MULTIPLICATIVE FUNCTIONS
}

\author{
P. ERDÖS
}

The present paper contains some results about the classical multiplicative functions $\phi(n), \sigma(n)$ and also about general additive and multiplicative functions.

(1) It is well known that $n / \phi(n)$ and $\sigma(n) / n$ have a distribution function. ${ }^{1}$ Denote these functions by $f_{1}(x)$ and $f_{2}(x)$. $\left(f_{1}(x)\right.$ denotes the density of integers for which $n / \phi(n) \leqq x$.) It is known that both $f_{1}(x)$ and $f_{2}(x)$ are strictly incrtasing and purely singular. ${ }^{1}$ We propose to investigate $f_{1}(x)$ and $f_{2}(x)$; we shall give details only in case of $f_{1}(x)$. First we prove the following theorem.

THeOREM 1. We have for every $\epsilon$ and sufficiently large $x$

(1) $\exp (-\exp [(1+\epsilon) a x])<1-f_{1}(x)<\exp (-\exp [(1-\epsilon) a x])$ where $a=\exp (-\gamma), \gamma$ Euler's constant.

We shall prove a stronger result. Put $A_{r}=\prod_{i=1}^{r} p_{i}, p_{i}$ consecutive primes. Define $A_{k}$ by $A_{k} / \phi\left(A_{k}\right) \geqq x>A_{k-1} / \phi\left(A_{k-1}\right)$. Then we have

$$
1 / A_{k}<1-f_{1}(x)<1 / A_{k}^{1-e} \text {. }
$$

First of all it is easy to see that Theorem 1 follows from (2), since from the prime number theorem we easily obtain that $\log \log A_{k}$ $=(1+o(1)) a x$, which shows that (1) follows from (2).

(2) means that the density of integers with $\phi(n) \leqq(1 / x) n$ is between $1 / A_{k}$ and $1 / A_{k}{ }^{1-9}$.

We evidently have for every $n \equiv 0\left(\bmod A_{k}\right), n / \phi(n) \geqq x$, which proves

$$
1 / A_{k} \leqq 1-f_{1}(x) .
$$

To get rid of the equality sign, it will be sufficient to observe that there exist integers $u$ with $u / \phi(u) \geqq x,\left(u, A_{k}\right)=1$, and that the density of the integers $n \equiv 0(\bmod u), n \neq 0\left(\bmod A_{k}\right)$ is positive. This proves the first part of (2). The proof of the second part will be much harder. We split the integers satisfying $n / \phi(n) \geqq x$ into two classes. In the first class are the integers which have more than $\left[\left(1-\epsilon_{1}\right) k\right]=r$ prime factors not greater than $B p_{k}$, where $B=B\left(\epsilon_{1}\right)$ is a large number. In

Received by the editors June 22, 1945, and, in revised form, January 28, 1946.

1 These results are due to Schönberg and Davenport. For a more general result see P. Erdös, J. London Math. Soc. vol. 13 (1938) pp. 119-127. 
the second class are the other integers satisfying $n / \phi(n) \geqq x$. It is easy to see that the number of integers of the first class does not exceed

$$
2^{\pi\left(B p_{k}\right)} / A_{r}=2^{o\left(p_{k}\right)} / A_{r}<1 / A_{k}^{1-\bullet}
$$

since $\pi\left(B p_{k}\right)=o\left(p_{k}\right)(\pi(x)$ denotes the number of primes not greater than $x)$, and from the prime number theorem $\log A_{r}>(1-\epsilon) p_{k}$ if $\epsilon_{1}$ is small.

Let now $n$ be any integer of the second class. A simple argument shows that

$$
\prod_{p \mid n}^{\prime}\left(1-\frac{1}{p}\right)<\prod_{t=r+1}^{k-1}\left(1-\frac{1}{p_{t}}\right)<1-\frac{c_{1} \epsilon_{1}}{\log p_{k}} .
$$

The prime indicates that the product is extended over the $p>B p_{k}$. The first inequality follows from the definition of $A_{k}$, and from the fact that $n$ is of the second class, the second inequality follows from the prime number theorem. Thus we have

$$
\sum_{p \mid n}^{\prime} \frac{1}{p}>\frac{c_{1} \epsilon_{1}}{\log p_{k}} .
$$

Denote now by $J_{t}$ the interval $\left(B^{t} p_{k}, B^{t+1} p_{k}\right), t=1,2, \cdots$. It follows from (4) that for every integer of the second class there exists some $t$ such that

$$
\sum_{p \mid n} \frac{1}{p}>c_{1} \frac{\epsilon_{1}}{2^{t} \log p_{k}}
$$

where in $\sum_{t}$ the summation is extended over the primes in $J_{t}$. Thus for some $t, n$ must divide more than

$$
c_{1} \epsilon_{1}\left(B^{t} / 2^{t}\right)\left(p_{k} / \log p_{k}\right)=B_{t}
$$

primes in $J_{t}$. The density of the integers satisfying (6), that is, the density of the integers of the second class, is less than

$$
\sum_{t=1}^{\infty}\left(\sum_{p \text { in } J_{t}} \frac{1}{p}\right)^{B_{t}} /\left[B_{t}\right] !<\frac{1}{\left[B_{t}\right] !}<e^{-2 p_{k}}<\frac{1}{A_{k}},
$$

that is, $\sum_{p \text { in }_{t}} 1 / p<1$ for large enough $k$ ( $B$ is independent of $k$ ), if $B=B\left(\epsilon_{1}\right)$ is large enough. Theorem 1 now follows from (3) and (7).

From Theorem 1 we easily obtain that

$$
\lim _{x \rightarrow \infty} \frac{1}{x} \sum_{n=1}^{x} \exp (\phi(n))
$$

exists. In fact we can also prove that for $\alpha<a$ 


$$
\lim _{x \rightarrow \infty} \frac{1}{x} \sum_{n=1}^{x} \exp (\exp (\phi(n))
$$

exists. For $\alpha>a$ the limit is infinite.

THEOREM 2.

$$
1 / A_{k}^{1+e}<1-f_{1}(x)<1 / A_{k}^{1-\ell} .
$$

We omit the proof since it is very similar to that of Theorem 1 .

THEOREM 3. Let $\epsilon \rightarrow 0$, then

$$
f_{1}(1+\epsilon)=(1+o(1)) a / \log \epsilon^{-1}, \quad f_{2}(1+\epsilon)=(1+o(1)) a / \log \epsilon^{-1} \text {. }
$$

We prove only the first statement since the proof of the second is essentially the same. Let $n$ be an integer with $n / \phi(n) \leqq 1+\epsilon$. Clearly $n$ does not divide any prime $p<\left(1-(1+\epsilon)^{-1}\right)^{-1}=\epsilon^{-1}+O(1)$. Thus

$$
f_{1}(1+\epsilon)<(1+o(1)) a / \log \epsilon^{-1} \text {. }
$$

Denote by $J_{t}$ the interval

$$
\left(4^{t-1}\left(1-(1+\epsilon)^{-1}\right)^{-1}, 4^{t}\left(1-(1+\epsilon)^{-1}\right)^{-1}\right) \text {. }
$$

If an integer $n \neq 0\left(\bmod p_{i}\right), p_{i}<\left(1-(1+\epsilon)^{-1}\right)^{-1}$, does not satisfy $n / \phi(n) \leqq 1+\epsilon$, then a simple computation shows that for some $t$ it must have at least $t$ prime factors in $J_{t}$. Thus the number of these integers does not exceed

$$
(1+o(1)) \frac{a}{\log \epsilon^{-1}} \sum_{t=1}^{\infty}\left(\sum_{p \operatorname{in} J_{t}} \frac{1}{p}\right)^{t} / t !=o\left(a / \log \epsilon^{-1}\right),
$$

which together with (8) proves Theorem 3.

It follows from Theorem 3 that $f_{1}^{\prime}(1)=\infty$. It would be easy to show that $f_{1}^{\prime}(n / \phi(n))=\infty$ for every $n$.

Denote by $f_{1}{ }^{\alpha}$ and $f_{2}{ }^{\alpha}$ the distribution functions of

$$
\prod_{p \mid n}\left(1-\frac{1}{p}\right)^{-\alpha} \text { and } \sum_{d \mid n} \frac{1}{d^{\alpha}}, \quad \alpha>0 .
$$

THEOREM 4.

$$
f_{1}^{(\alpha)}(1+\epsilon)=(1+o(1)) \frac{a \alpha}{\log \epsilon^{-1}}, \quad f_{2}^{(\alpha)}=(1+o(1)) \frac{a \alpha}{\log \epsilon^{-1}} .
$$

We omit the proof since it is very similar to that of Theorem 3 .

Let us denote by $F_{\alpha}(x), \alpha>0$, the distribution function of $\prod_{p \mid n}\left(1-1 / \log p^{\alpha}\right)^{-1}, \alpha>0$. 
TheOREM 5.

$$
F_{1}(1+\epsilon)=(1+o(1)) b \epsilon,
$$

that is, $F_{1}^{\prime}(1)=b$. Also $F_{\alpha}^{\prime}(1)=0$ for $\alpha<1$ and $F_{1}^{\prime}(1)=\infty$ for $\alpha>1$.

We do not give the details of the proof since it would be long and similar to that of Theorem 3. We just make the following remarks: If $n$ satisfies

$$
\sum_{p \mid n} \frac{1}{\log p} \leqq 1+\epsilon
$$

then $n$ does not divide any prime $p \leqq \exp (1 / \epsilon)$. Thus $F_{1}^{\prime}(1+\epsilon)$ $\leqq(1+o(1)) a \epsilon$. But here (unlike in Theorem 3 ) we have $F_{1}(1+\epsilon)$ $=(1+o(1)) b, b<a$. We obtain analogous results if we consider the additive function $\sum_{p \mid n} 1 / \log$ p. It is possible that $F_{1}^{\prime}(x)$ exists for every $1 \leqq x$, but this we can not prove.

(2) The following results are well known:

$$
\sum_{m=1}^{x} \frac{\phi(m)}{m}=(1+o(1)) \frac{6}{\pi^{2}} x, \quad \sum_{m=1}^{x} \frac{\sigma(m)}{m}=(1+o(1)) \frac{\pi^{2}}{6} x .
$$

The density of integers for which $\sigma(n+1) /(n+1)>\sigma(n) / n$ is $1 / 2$, also the density of integers for which $\phi(n+1) /(n+1)>\phi(n) / n$ is $1 / 2.2$ Now we prove the following theorem.

Theorem 6. Let $g(n) / \log \log \log n \rightarrow \infty$. Then we have

$$
\sum_{m=n}^{n+g(n)} \frac{\phi(m)}{m}=(1+o(1)) \frac{6}{\pi^{2}} g(n) .
$$

(ii) The number of integers $m$ in $(n, n+g(n))$ which satisfy $\phi(m+1) /(m+1)>\phi(m) / m$ equals $(1+o(1)) g(n) / 2$.

(iii) The number of integers $m$ in $(n, n+g(n))$ which satisfy $m / \phi(m) \leqq c$ equals $(1+o(1)) g(n) f_{1}(c)$. In other words the distribution function of $\phi(m) / m$ in $(n, n+g(n))$ is the same as the distribution function of $\phi(m) / m$.

All these results are best possible; they become false if for infinitely many $n, g(n)<c \log \log \log n$.

We prove only (i); the proof of (ii) and (iii) are similar. Let $A=A(n)$ tend to infinity sufficiently slowly. Put

$$
\frac{\phi(m)}{m}=D_{1}(m) D_{2}(m),
$$

${ }^{2}$ P. Erdös, Proc. Cambridge Philos. Soc. vol. 32 (1936) pp. 530-540. 
where

$$
D_{1}(m)=\prod_{p \mid m}^{\prime}\left(1-\frac{1}{p}\right), \quad D_{2}(m)=\prod_{p \mid m}^{\prime \prime}\left(1-\frac{1}{p}\right) .
$$

The prime indicates that $p \leqq A$, the two primes that $p>A$. We evidently have

$$
\begin{aligned}
\sum_{m=n}^{n+g(n)} \frac{\phi(m)}{m} & <\sum_{m=n}^{n+o(n)} D_{1}(m)=\sum_{a}^{\prime \prime \prime}\left(\frac{g(n)}{d}\right) \frac{\mu(d)}{d} \\
& =(1+o(1)) g(n) \prod_{p \leqq A}\left(1-\frac{1}{p^{2}}\right)=(1+o(1)) \frac{\pi^{2}}{6} g(n)
\end{aligned}
$$

where the three primes indicate that the prime factors of $d$ are not greater than $A$, and $(g(n) / d)$ denotes the number of multiples of $d$ in $(n, n+g(n))$. Now we show that for sufficiently large $A$ the number of integers in $(n, n+g(n))$ which satisfy

$$
D_{2}(m)<1-\epsilon
$$

is $o(g(n))$. It will be sufficient to show that

$$
\prod_{m} D_{2}(m)>(1-\eta)^{o(n)}
$$

for every $\eta>0$, the product over $m$ runs in $(n, n+g(n))$. We evidently have

$$
\prod_{m} D_{2}(m)>\Pi_{1}\left(1-\frac{1}{p}\right)^{2 g(n) / p-1} \Pi_{2}\left(1-\frac{1}{p}\right)
$$

where, in $\prod_{1}, A<p \leqq g(n)$, and in $\prod_{2}, p$ runs through the prime factors greater than $g(n)$ of $n(n+1) \cdots(n+g(n))$. Clearly

$$
\prod_{1}>\prod_{p>A}\left(1-\frac{c}{p^{2}}\right)^{o(n)}>\left(1-\eta_{1}\right)^{o(n)} \text {. }
$$

From the prime number theorem we have $\prod_{p \leqq x} p<e^{2 x}$. Thus

$$
\Pi_{2}>\prod_{p \leqq 2 y}\left(1-\frac{1}{p}\right)>\frac{c_{1}}{\log y}
$$

where $y=\log [n(n+1) \cdots(n+g(n))]$. Hence using $g(n) / \log \log \log n$ $\rightarrow \infty$, we obtain by a simple calculation that

$$
\prod_{2}>\left(1-\eta_{2}\right)^{g(n)}
$$

which proves (11) and therefore (10). From (9) and (10) we obtain by a simple argument that 


$$
\sum_{m=n}^{n+g(n)} \frac{\phi(m)}{m}>(1-o(1)) \sum_{m=n}^{n+g(n)} D_{1}(m)=(1+o(1)) g(n) \frac{\pi^{2}}{6} .
$$

(i) now follows from 9 and (12). ${ }^{3}$

Now we are going to prove that (i) is best possible. Put $g(N)$ $=c \log \log \log N, n / 2<N<n$. Further let $A_{1}, A_{2}, \cdots, A_{r}$, $r=\left[2^{-1} \log \log \log n\right]$ be relatively prime integers all of whose prime factors are less than $2^{-1} \log n$ and for which

$$
1 / 4<\phi\left(A_{i}\right) / A_{i}<1 / 2, \quad i=1,2, \cdots, r .
$$

This is obviously possible since

$$
\prod_{p<(\log n) / 2}\left(1-\frac{1}{p}\right)<\frac{c}{\log \log n}<\left(\frac{1}{4}\right)^{(\log \log \log n) / 2} .
$$

Now choose $n / 2<N<n$ so that $N+j \equiv 0\left(\bmod A_{j}\right), j \leqq r$. This is possible since by the prime number theorem $A_{1} \cdot A_{2} \cdots A_{r}<n / 2$. (In all cases where we refer to the prime number theorem a more elementary result would be sufficient.) Clearly

$$
N+(\log \log \log n) / 2 \frac{\phi(m)}{m}<\frac{\log \log \log n}{4} .
$$

From (9) we have

$$
\sum_{N+(\log \log \log n) / 2}^{N+g(N)} \frac{\phi(m)}{m}<(1+o(1)) \frac{6}{\pi^{2}}\left(g(N)-\frac{\log \log \log n}{2}\right) .
$$

Thus finally from (10) and (11) we obtain by a simple calculation

$$
\sum_{m=N}^{N+o(N)} \frac{\phi(m)}{m}<(1-c) \frac{6}{\pi^{2}} g(N),
$$

which shows that (i) is best possible. ${ }^{4}$

ThEOREM 7. Let $g_{1}(n) / \log \log n \rightarrow \infty$. Then we have

$$
\sum_{m=n}^{n+o_{1}(n)} \frac{\sigma(m)}{m}=(1+o(1)) \frac{\pi^{2}}{6} g_{1}(n) \text {. }
$$

(ii) Let $g_{2}(n) / \log \log \log n \rightarrow \infty$. The number of integers $m$ in $\left(n, n+g_{2}(n)\right)$ which satisfy $\sigma(n+1) /(n+1)>\sigma(n) / n$ equals $(1+o(1))$ $\cdot g(n) / 2$.

${ }^{8}$ This proof is similar to a proof in P. Erdös, J. London Math. Soc. vol. 10 (1935) pp. 128-131.

4 This proof is similar to a proof of Chowla and Pillai, J. London Math. Soc. vol. 5 (1930) pp. 95-101. 
(iii) The number of integers $m$ in $(n, n+g(n))$ which satisfy $\sigma(m) / m$ $<c$ equals $(1+o(1)) g(n) f_{2}(c) \cdot{ }^{5}$ All these results are best possible.

We omit the proof of Theorem 7, since it is similar to that of Theorem 6 . We must allow $g_{1}(n) / \log \log n \rightarrow \infty$, since it is well known that for some $m \leqq n, \sigma(m)>c \log \log n$ (for example, $m=\prod_{p<(\log n) / 2} p$ ).

Let $f(n) \leqq 1$ and $F(n) \geqq 1$ be multiplicative functions with

$$
\sum_{p} \frac{1-f(p)}{p}<\infty \text { and } \sum_{p} \frac{F(p)-1}{p}<\infty .
$$

Then we have:

TheOREM 8. Let $A=A(n)$ tend to infinity arbitrarily slowly, then

and

$$
\frac{1}{A} \sum_{m=n}^{n+A} f(m)<\left(1+(o(1)) \frac{1}{n} \sum_{m=1}^{n} f(m)\right.
$$

$$
\frac{1}{A} \sum_{m=n}^{n+A} F(m)>(1+o(1)) \frac{1}{n} \sum_{m=1}^{n} F(m) .
$$

The proof is quite trivial; it is similar to that of (9). It can be shown that $\lim (1 / n) \sum_{m=1}^{n} f(m)$ and $\lim (1 / n) \sum_{m=1}^{n} F(m)$ exist.

Denote by $V(n)$ the number of prime factors of $n$ and by $d(n)$ the number of divisors of $n$. We can prove analogs to Theorem 6 for these functions. But the results are very unsatisfactory since for $v(n)$ we have to choose $g(n)=n^{\epsilon / \log \log n}$ and for $d(n), g(n)=n^{\circ}$ for some suitable $c$. These results are probably very far from best possible.

(3) Let $n=p_{1}^{\alpha_{1}} p_{2}^{\alpha_{2}} \cdots p_{k}^{\alpha_{k}}, p_{1}^{\alpha_{1}}<p_{2}^{\alpha_{2}}<\cdots<p_{k}^{\alpha_{k}}$. Put $\left(p_{i}^{\alpha_{i}}\right)^{b_{i}}$ $=p_{i}{ }^{\alpha i+1}$. We prove the following theorem.

THEOREM 9. Let $1<x$, then for almost all $n$ the number of b's greater than $x$ equals

$$
x^{-1} \log \log n+o(\log \log n) \text {. }
$$

REMARK. We immediately obtain that every interval $(x, x+\epsilon)$ contains $(1+o(1))(\epsilon / x(x+\epsilon)) \log \log n b$ 's.

We are going to give only an outline of the proof. First of all we can assume that all the $\alpha$ 's are 1, since for large $r$ the number of integers not greater than $n$ for which $r$ or more of the $\alpha$ 's is greater than 1 is less than $\epsilon n$, since the number of these integers is clearly less than

$$
\left(\sum_{p} \frac{1}{p^{2}}\right)^{r} / r !<\epsilon n .
$$

- This result has been stated previously, see footnote 4. 
Denote by $F(n)$ the number of prime factors $p$ of $n$ such that no prime $q$ in $\left(p, p^{x}\right)$ divides $n . F(n)$ is thus the number of $b$ 's not less than $x$. We have

$$
\sum_{m=1}^{n} F(m)=\frac{1}{x} \log \log n+o(\log \log n) .
$$

We now give a sketch of the proof. Clearly

$$
\sum_{m=1}^{n} F(m)=\sum_{p} f_{p}(n)
$$

where $f_{p}(n)$ denotes the number of integers $m \leqq n$, with $m \equiv 0(\bmod p)$ and $m \neq \equiv 0(\bmod q), p<q<p^{x}$. It is easy to see that for $p<n^{e}$

Also for all $p$

$$
f_{p}(n)=(1+o(1)) n / p x \quad(p \text { large }) .
$$

$$
f_{p}(n) \leqq n / p \text {. }
$$

Thus

$$
\begin{aligned}
\sum_{m=1}^{n} F(m) & =\sum_{p \leqq n^{e}} \frac{n}{p x}+O \sum_{n^{\epsilon}<p<n} \frac{n}{p}+o(\log \log n) \\
& =(1+o(1)) \frac{\log \log n}{x},
\end{aligned}
$$

which proves (14). Now we have to show that

$$
F(m)=(1+o(1))(\log \log n) / x
$$

for almost all $m \leqq n$. We use Turán's method. ${ }^{6}$ We have

$$
\begin{aligned}
\sum_{m=1}^{n}\left(F(m)-\frac{1}{x}\right. & \log \log n)^{2} \\
& =\sum_{m=1}^{n} F^{2}(m)-\frac{2}{x} \log \log n \sum_{m=1}^{n} F(m)+n\left(\frac{\log \log n}{x}\right)^{2} .
\end{aligned}
$$

Now

$$
\sum_{m=1}^{n} F^{2}(m)=(1+o(1)) n\left(\frac{\log \log n}{x}\right)^{2} .
$$

We omit the proof of (15), it is similar to the proof of (14). Thus

$$
\sum_{m=1}^{n}\left(F(m)-\frac{1}{x} \log \log n\right)^{2}=o\left(n(\log \log n)^{2}\right)
$$

which proves Theorem 9 .

' P. Turán, J. London Math. Soc. vol. 9 (1934) pp. 274-276. 
TheOREM 10. For almost all $n$ we have

$$
\sum_{p_{i} \mid n} b_{i}=(1+o(1)) \log \log n \log \log \log n .
$$

THEOREM 11. Let $1<x$ be any number. For almost all $n$ there exist intervals $\left(m, m^{x}\right), m^{x} \leqq n$, such that for every $m \leqq y \leqq m^{x}, n \neq 0(\bmod y)$.

We omit the proofs of Theorems 10 and 11. They are similar to that of Theorem 9.

For some time I have not been able to decide the following question: Is it true that almost all integers $n$ have divisors $d_{1}$ and $d_{2}$, such that $d_{1}<d_{2}<2 d_{1}$.

(4) Let $f(n)$ be an additive function which has a distribution function. Then it is well known that ${ }^{7}$

$$
\sum_{p} \frac{f(p)^{\prime}}{p}<\infty, \quad \sum_{p} \frac{\left(f(p)^{\prime}\right)^{2}}{p}<\infty,
$$

$f(p)^{\prime}=f(p)$ if $|f(p)| \leqq 1$ and $f(p)^{\prime}=1$ if $|f(p)|>1$. Assume now that $\left|f\left(p^{\alpha}\right)\right| \leqq C(f(n)$ is assumed to be real valued). We prove the following theorem.

THEOREM 12. Let $\left|f\left(p^{\alpha}\right)\right| \leqq c$. Denote by $F(x)$ the distribution function of $f(x)$. We have

$$
F(x)>1-\exp (-c x),
$$

for every $c$ and sufficiently large $x$. In other words the density of integers with $f(n) \geqq x$ is less than $\exp (-c x)$.

Put $g(n)=\exp (2 c f(n)), g(n)$ is multiplicative and clearly has a distribution function. Define

$$
f_{k}(n)=\sum_{p \mid n, p \leqq k} f(p), \quad g_{k}(n)=\exp \left(2 c f_{k}(n)\right) .
$$

For sake of simplicity we assume that $f\left(p^{\alpha}\right)=f(p)$. It is well known that the distribution function $F_{k}(x)$ of $f_{k}(n)$ converges to $F(x)$, thus the distribution function $G_{k}(x)$ of $g_{k}(x)$ converges to $G(x)(G(x)$ is the distribution function of $g(x))$. Suppose now that Theorem 12 is false, then there exists a constant $c$ and infinitely many $x_{r}$ with $x_{r} \rightarrow \infty$ and

$$
F\left(x_{r}\right)>1-\exp \left(-c x_{r}\right) .
$$

Therefore for any $r$ there exists a $k$ so large that

$$
F_{k}\left(x_{r}\right)>1-\exp \left(-c x_{r}\right) \text {. }
$$

\footnotetext{
7 P. Erdös and A. Wintner, Amer. J. Math. vol. 61 (1939) pp. 713-721.
} 
Thus the density of integers with $g_{k}(n)>\exp \left(2 c x_{r}\right)$ is greater than $\exp \left(-c x_{r}\right)$ and hence

$$
\sum_{m \leqq n} g_{k}(m)>(1-\epsilon) \exp \left(c x_{r}\right) \cdot n
$$

for $n$ sufficiently large. Thus for any $A$ there exists $k$ and $n_{0}$, such that for all $n>n_{0}$

On the other hand

$$
\sum_{m \leqq n} g_{k}(m)>A n
$$

$$
\sum_{m \leqq n} g_{k}(m)=\sum_{m=1}^{n} \prod_{p \mid m} g_{k}(p)=\sum_{m=1}^{n} \prod_{p \mid m}\left(1+\left(g_{k}(p)-1\right)\right) .
$$

Put $g_{k}(p)-1=h_{k}(p)$. Clearly

$$
\sum_{m=1}^{n} g_{k}(m)=\sum_{m=1}^{n} \prod_{p \mid m}\left(1+h_{k}(p)\right)=\sum_{d}\left[\frac{n}{d}\right] h_{k}(d)
$$

where $h_{k}(d)=\prod_{p \mid d} h_{k}(p)$. Thus

$$
\sum_{m=1}^{n} g_{k}(m) \leqq n \sum_{d} \frac{h_{k}(d)}{d}=n \prod_{p}\left(1+\frac{h_{k}(p)}{p}\right) .
$$

From the fact that $g(n)$ has a distribution function and that $f\left(p^{\alpha}\right)$ is bounded, it easily follows that (we shall give the details in the proof of Theorem 13)

$$
\sum_{p} \frac{h(p)}{p}<\infty, \quad \sum_{p} \frac{(h(p))^{2}}{p}<\infty, \quad h(p)=g(p)-1 .
$$

Thus finally

$$
\sum_{m=1}^{n} g_{k}(m)<c_{1} n \prod_{p}\left(1+\frac{h(p)}{p}\right)<c_{2} n,
$$

which contradicts (17), and this contradiction establishes the theorem.

It is easy to see that Theorem 12 is best possible. Let $\phi(x)$ tend to infinity arbitrarily slowly; then there exists an additive function $f(n)$ such that its distribution function $F(x)$ satisfies $F\left(x_{i}\right)<1-\exp \left(-\phi\left(x_{i}\right) x_{i}\right)$ for an infinite sequence $x_{i}$ with $x_{i} \rightarrow \infty$. We omit the proof.

Theorem 13. Let $g(n) \geqq 0$ be multiplicative. Then the necessary and sufficient condition for the existence of a distribution function is that 


$$
\sum_{p} \frac{(g(p)-1)^{\prime}}{p}<\infty, \quad \sum_{p} \frac{\left((g(p)-1)^{\prime}\right)^{2}}{p}<\infty,
$$

where $(g(p)-1)^{\prime}=g(p)-1$ if $|g(p)-1| \leqq 1$ and 1 otherwise.

The proof follows very easily from (16). Put $\log (g(n))=f(n) . g(n)$ has a distribution function if and only if $f(n)$ has a distribution function. Thus from (16)

$$
\sum_{p} \frac{(\log g(p))^{\prime}}{p}<\infty, \quad \sum_{p} \frac{\left((\log g(p))^{\prime}\right)^{2}}{p}<\infty .
$$

Now it follows from (19) that if we neglect a sequence of primes $q$ with $\sum 1 / q<\infty$ that $|g(p)-1|<1 / 2$. Thus

$\log g(p)=\log (1+(g(p)-1))=g(p)-1+(1 / 2)(g(p)-1)^{2}+\cdots$.

Also simple computation shows that $(\log g(p))^{1}>(1 / 4)(g(p)-1)^{2}$. Thus from (19)

and

$$
\sum_{p} \frac{(g(p)-1)^{2}}{p}<\infty
$$

$$
\sum_{p}\left((1 / 2)(g(p)-1)^{2}+(g(p)-1)^{3}+\cdots\right)<\infty .
$$

Thus $\sum_{p}(g(p)-1) / p<\infty$, which shows that (18) is necessary.

If the two series in (18) converge, then clearly

$$
\sum_{p} \frac{\log g(p)}{p}=\sum_{p}\left(\frac{(g(p)-1)}{p}+\frac{(1 / 2)(g(p)-1)^{2}}{p}+\cdots\right)<\infty
$$

and

$$
\sum_{p} \frac{(\log g(p))^{2}}{p}<c \sum_{p} \frac{(g(p)-1)^{2}}{p}<\infty,
$$

which shows that $f(n)$, and therefore $g(n)$, has a distribution function. Thus (18) is necessary, which completes the proof of Theorem 13.

These results suggest that if $g(n)$ is multiplicative, satisfies (18), $\left|g\left(p^{\alpha}\right)\right|<c$, then $g(n)$ has a mean value, that is, $\lim (1 / x) \sum_{n-1}^{x} f(n)$ exists. I have not yet been able to prove this.

UNIVERSITY OF MICHIGAN 